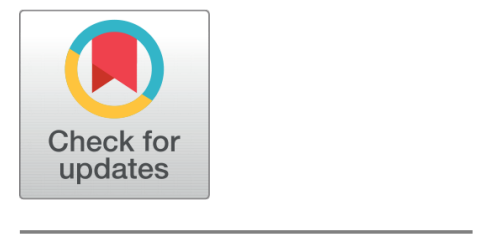

open ACCess

Received: 11.05.2021

Accepted: 13.08.2021

Published: 13.09 .2021

Citation: Gupta R, Kumar J (2021) Outlier based Human Fall Detection using One-Class Classification. Indian Journal of Science and Technology 14(30): 2494-2503. https ://doi.org/10.17485/IJST/v14i30.821

* Corresponding author.

rashmi.kvk@gmail.com

Funding: None

Competing Interests: None

Copyright: (c) 2021 Gupta \& Kumar. This is an open access article distributed under the terms of the Creative Commons Attribution License, which permits unrestricted use, distribution, and reproduction in any medium, provided the original author and source are credited.

Published By Indian Society for Education and Environment (iSee)

ISSN

Print: 0974-6846

Electronic: 0974-5645

\section{Outlier based Human Fall Detection using One-Class Classification}

\author{
Rashmi Gupta ${ }^{1 *}$, Jeetendra Kumar ${ }^{1}$ \\ 1 Assistant Professor, Atal Bihari Vajpayee University, Bilaspur (C.G.), India
}

\section{Abstract}

Objective: This work aims to develop a human fall detection method that is trained using data of routine movement of people only collected from accelerometer sensor to stay away from irregular fall detection model. This work also aims to analyze the effect of calculated features on the fall detection model. Methodology: In the proposed method, The fall detection model is built using one-class classification. At first, data of accelerometer sensor in three directions has been used to detect the fall events. Five feature vectors i.e. resultant, variance, standard deviation, Root Mean square, and Euclidean Norm have been calculated. These four features along with one class SVM have been used to build fall detection model in ways (1) Using only resultant features only (2) Using all calculated features. This model was trained using only activities of daily living (ADL) and tested on both daily living activities and fall activities. Findings: It is found that when the model was built using all calculated features, the sensitivity was $100 \%$ and specificity was $94.92 \%$ when mobile is in the pocket and; sensitivity was $100 \%$ and specificity was $93.61 \%$ when mobile is in handbag which is better than when the model was built using resultant features only. We have also studied the effect of individual features on this fall detection model and it is found that variance played a very important role to classify fall activities and ADL activities. Novelty - The proposed fall detection method is built using one-class classification so that the proposed model will be reliable to detect falls in real life. In the proposed work, the effect of features on the fall detection model is also analyzed.

Keywords: One class classification; accelerometer; outlier detection; fall detection; Bagging classifier

\section{Introduction}

Human falls are a major health issue for the elderly. Falls occur in $13 \%$ of the population over the age of 65 , and $13 \%$ of this group lives alone. Falls and instability are common causes of death ${ }^{(1)}$. Human falls can be defined as" to suddenly go down onto the ground or towards the ground without intending to or by accident". Fall detection systems detect the human fall and inform the caretaker that the person has been fallen and needs immediate help. According to a WHO fact sheet on falls reviewed in September 2016, "falls are the second leading cause of accidental or unintentional injury deaths 
worldwide and each year, an estimated 424,000 people die as a result of falls around the world, with more than 80 percent of these deaths occurring in low- and middle-income countries" (2). Falls not only cause moderate to severe injuries to the elderly, but they also place a mental and financial burden on the elderly and their relatives. When confronted with this situation, it is critical to detect elderly falls and provide emergency assistance as soon as possible. In a nutshell, those who fall and are unable to summon help must be found and treated as soon as possible.

The fall detection market is divided into three segments. It can be divided into wearable and non-wearable sensor segments based on the various types of sensors. Wearable sensors are easy to use, lightweight, and easily portable from one place to other whereas non-wearable sensors are fitted in the room, corridor, or other outdoor areas. If we consider technology, then the system market can be divided into GPS systems, mobile phones, and sensor-based market segments. By considering end-user fall detection, the market can be segmented into home care and senior assisted living facilities.

There are many important factors of fall detection

1. The human fall detector helps to reduce the time of lying on the floor after falling. This is the key factor to reduce the severity of falls.

2. Fall detection reduces the fear of falling in the elders. They have confidence that if they fall unconsciously anytime then a message of their falling will be immediately sent to their caretaker.

3. Fall detection reduces medical expenses because fall detection reduces the severity of falls.

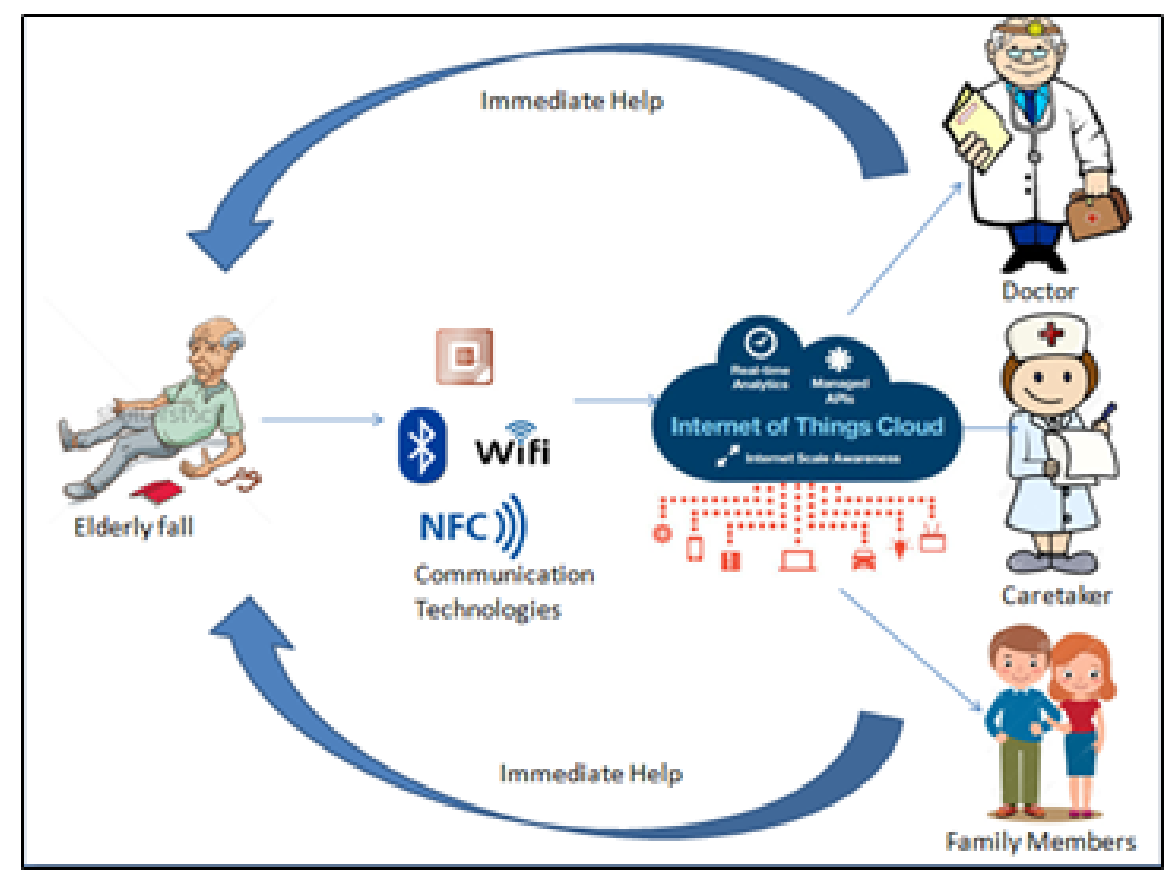

Fig 1. General Fall detection scenario

Fall detection can also be implemented on the Internet of Things (IoT) environment as shown in Figure 1 because sometimes fall detection algorithms are very complex and it is very costly to run fall detection algorithms in the smartphone. When we use the Internet of Things environment, accelerometer sensor data is transferred to the cloud where complex machine learning algorithms are used to analyze whether the fall event has occurred or not, and information of falling is immediately sent to the doctor, caretaker, or family members. Various methods ${ }^{(3,4)}$ have implemented fall detection using the internet of things.

Fall detection methods can be divided into various categories based on the different types of technologies used. According to ${ }^{(5)}$, falls can be divided into the following categories.

1. Wearable sensors based fall detection

2. Ambient sensors based fall detection

3. Vision-based fall detection 
Various categories of human fall detection methods have been shown in Figure 2 In wearable sensor-based fall detection, sensors can be worn like a pendant, wristband, armband, etc. Various types of sensors are used in wearable fall detection technologies like accelerometers, gyroscopes, magnetometers, etc. Ambient sensor-based approaches are somewhat different from wearable sensors. Ambient sensor-based approaches use the sound detector, pressure detector, vibration detector, etc for sensing fall. Vision-based approaches are based on analyzing images or videos of a falling person. Image processing techniques are mainly used in vision-based fall detection category. Devices that are used in vision-based fall detection techniques are cameras, webcam and Microsoft Kinect cameras, etc. Vision-based techniques are considered as most non-obtrusive fall detection methods.

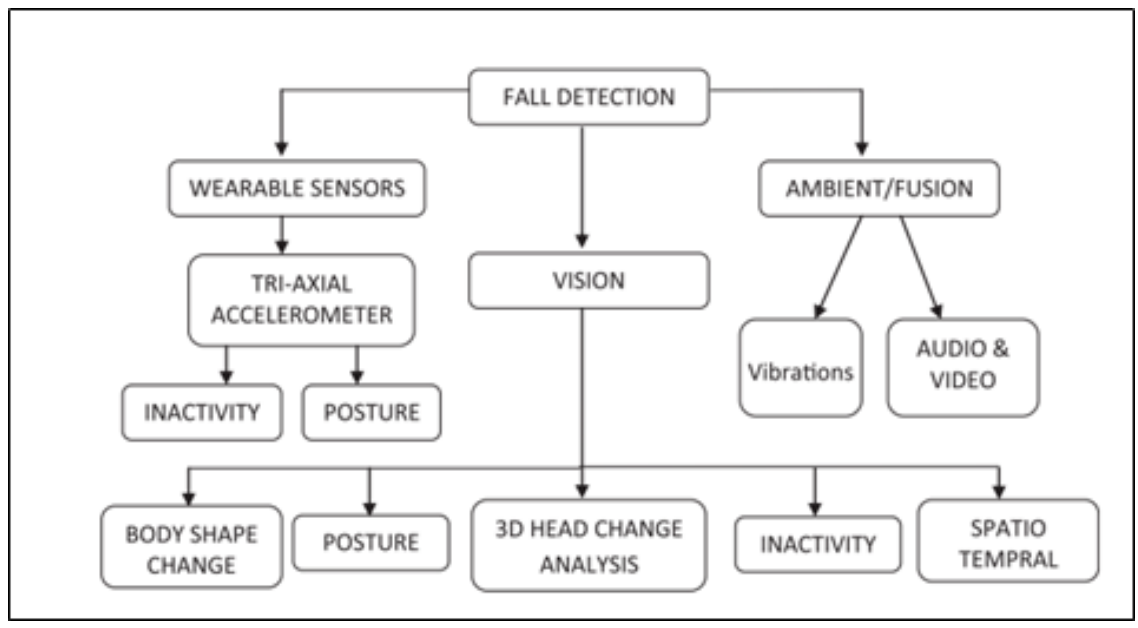

Fig 2. Categories of human fall detection methods ${ }^{(1)}$

Binary classification is a classification method that divides data into two groups: positive and negative. The scenario of positive and negative classes gives rise to the concept of one-class classification. In a one-class classification, Generally data of negative class is available in very less amount. As a result, the problem of one-class classification is more difficult than the problem of conventional multi-class / binary classification. Therefore, one-class classification algorithms should be expected to require a greater number of training instances than traditional multi-class classification algorithms ${ }^{(6)}$.

One class classification method is divided into two approaches the first approach is based on the availability of training data and the second approach is based on the type of algorithm used as shown in Figure 3. Algorithms can be one-class support vector machines (OSVM) and non-one-class support vector machines (Non- OSVM). According to Shehroz S. Khan et.al. ${ }^{(7)}$, one class classification methods can be applied in various domains like text/doccument classification, identification of abnormal behavior of machine, disaster activity identification etc.

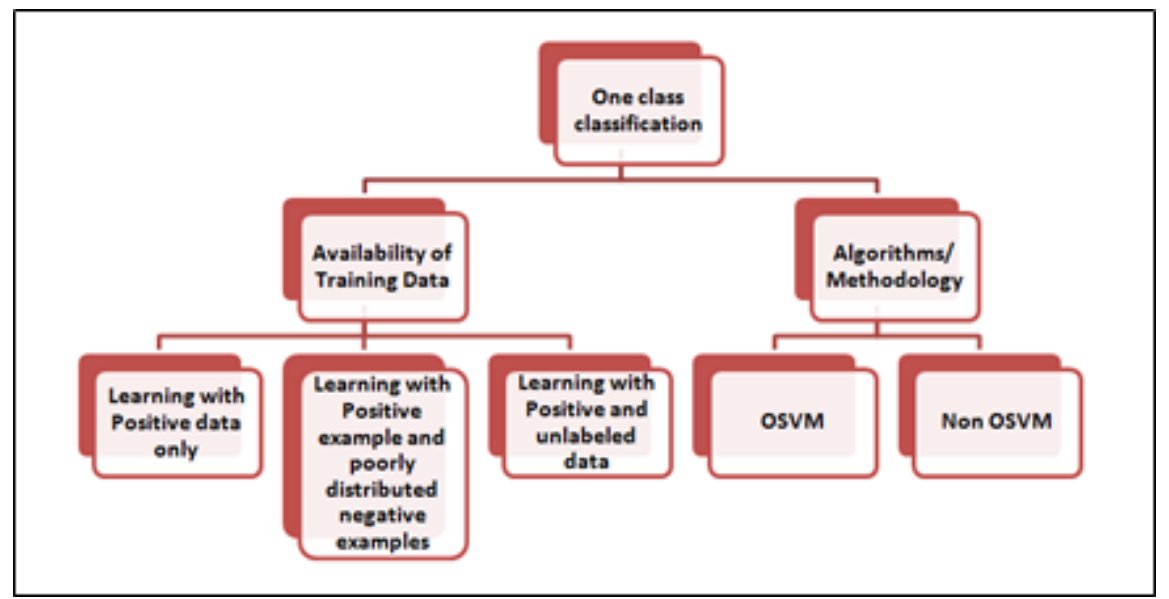

Fig 3. Categories of One class classification 
It is relevant to the case where we have to classify whether the machine, operating normally or not. In this case, the dataset of the normal behavior of the machine is available but fault occurs very less so data of fall activities are available in very fewer numbers. Most of the time dataset of abnormal behavior of the machine is not available. In this type of case, one class classifier classifies the normal behavior of machines in one class, and behavior other than normal behavior is considered as outlier. One class classification is also known as outlier detection, novelty detection. The term one class classifier was firstly coined by Minter (1975). In a Bayes classification learning context he had been the first to use the term 'one class classification' four decades ago and only labeled 'class of interest' data is required. After that Moya et al. ${ }^{(8)}$ formally used the term "One-Class Classification" in their research work. Similar ideas, such as Outlier Detection ${ }^{(9)}$, Novelty Detection ${ }^{(10)}$, or Concept Learning ${ }^{(11)}$ were subsequently used by different researchers.

We have used one-class classification in our proposed method of fall detection because, in real-life scenarios, data of normal daily activities are easily available but data of fall activities are rarely available because fall activities in elder persons are normally not recorded or not available. Two different predictions are possible in one classification, which means an instance belongs to the studied class during training and unknown in which the instance does not seem to be part of the previous class ${ }^{(12)}$. In this approach, we have considered normal daily activities as targets and, activities other than normal activities are considered as fall(outlier).

\section{Literature Review}

To analyze the sensor signal pattern of human fall detection, much research works have been carried out. In $2017^{(13)}$ has given the review of pervasive computing for fall detection. Methods which detect human falls automatically can be categorized as (1) Thresholding-based approaches (2) Machine Learning approaches. Thresholding-based approaches are lightweight and consume less processing time whereas machine learning approaches are very complex and need high processing time. A lot of research has been done in both areas. In thresholding-based approaches, various methods ${ }^{(14-18)}$ have been proposed. Even though machine learning algorithms are very complex but using these algorithms better results have been obtained. Research papers ${ }^{(16)}$, (19-26) used machine learning-based approaches for fall detection. Machine learning algorithms performed very well as compared to threshold-based methods.

Our method is based on machine learning but used one-class classifier instead of a multiclass or binary classifier. In various applications such as smart home ${ }^{(27,28)}$, security ${ }^{(29-31)}$, image processing ${ }^{(32)}$, one classification was applied. In 2016 Kemmeler et.al ${ }^{(33)}$ presented a paper "One-class classification with Gaussian processes". They used Gaussian process-based one-class classification methods GP-Reg-M and GP- Reg. But GP-Reg-M and GP- Reg. were not preferable applications anywhere because they are highly application dependant. In 2013 leng et al. ${ }^{(34)}$ presented a paper that is based on the combination of one-class classification and extreme machine learning (EML). They proposed a one-class classifier-based extreme learning machine to tackle the slow learning speed in the auto encoder neural network (ELM). The ELM was necessary because the hidden layer does not need to be tuned and final weight can be determined analytically, they concluded that extreme machine learning for a particular classification was effective.

Some fall detection methods ${ }^{(35-37)}$ have employed one class classification for detecting falls. In the area of fall detection, medrano et.al. ${ }^{(35)}$ used one-class classification in their proposed method. They recorded a dataset of ten volunteers. They used support vector machine (SVM) and (K nearest neighbor) KNN classifiers to train the fall detection model. The particular record was classified as fall if the threshold value was less than the novelty score. They also personalized their method. Out of data of 10 persons, they train the model using one person's data and tested that model on all other person's data. In 2016, Medrano et al. ${ }^{(36)}$ upgraded their method for detecting human falls which was based on combining the novelty detectors. In this paper, they calculated four features (i.e. $\mathrm{dNN}, \Delta$, vf, D) and used two combination rules which were based on probability and output labels. Instead of a single novelty detector, they combine the novelty detectors. They achieved 0.979 sensitivity and 0.967 specificity with a combination of novelty detectors and; 0.964 sensitivity and 0.955 specificity in SVM-based fall detection. In 2015 Daniela et al. presented a paper "Falls as anomalies? An experimental evaluation using smartphone accelerometer data" (37). In their experiment two publically available datasets ${ }^{(22,25)}$ were used in combination with one class classification. After calculation four features i.e. raw data, magnitude, accelerometer features, local temporal patterns they evaluate 1 class KNN, 2 class KNN, 1 class SVM, and 2 class SVM. They found that 2 class SVM-based fall detection method outperformed others. They got 0.927 sensitivity 0.898 specificity in one class KNN, 0.934 sensitivity 0.936 specificity in two-class KNN method, 0.926 sensitivity 0.879 specificity in one-class SVM, and 0.961 sensitivity 0.968 specificity in two-class SVM classifiers. However, while detecting reallife falls, two classification-based fall detection results decrease substantially because in two class classfication fall data which is simulated in the lab have been used for training and in one class classification only ADL data have been used for training. In 2021 sofia et al. ${ }^{(38)}$ proposed wheelchair fall detection system using one-class support vector machine. Using accelerometer 
and gyroscope data with ReliefF algorithm to select best features, they achieved 96\% g-mean score.

\section{Methodology}

In the proposed method bagging-based one-class classification for fall detection has been used. In real life real fall detection data is not available and data of normal daily activities are easily available and can be easily recorded. This is the reason behind one class classification-based method. This method considers daily living activities as targets and activities other than daily activities are considered as outlier i.e. fall activities.

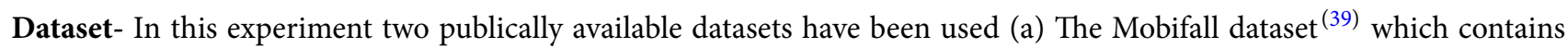
simulated fall and ADL activity performed by 31 persons (17 males and 14 females). The age of persons varies from 24 to 47. These subjects perform 9 ADL activities standing, walking, jogging, jumping, stairs up, stairs down, sit chair, car step in, car step out, and four fall activities (Forward lying (FOL), Front knee lying (FKL), Back sitting chair (BSC), Sideward lying (SDL). Each person did these activities three times. They collected data from three sensors accelerometer, gyroscope, and orientation sensor. We have used only accelerometer data. This dataset does not remove the gravity component from accelerometer data. (b) Other datasets medrano et al. ${ }^{(35)}$ contain a wide range of daily living activities and fall activities. It is sufficient for building any suitable fall detection model. Eight different types of simulated falls were performed by participants. Falls in a laboratory environment were completed on a soft mattress. During the fall events participants carried the smartphone in their both pockets. There were 503 fall event were recorded. The ADL study was conducted in real life. For at least one week, participants had a smartphone in their pocket to record their daily behaviour. Each subject has obtained an average of 800 records of ADL activities. Each ADL or fall is turned into a recording vector, consisting of 51 values of acceleration at $50 \mathrm{~Hz}$ and centred at the peak, which we call in this paper. Each ADL was or fell into a 51-value vector.

Features Extraction- Five different features have been calculated on this dataset to make an effective model for fall detection.

1. Resultant- We have calculated the resultant of accelerometer values in $x, y$, and $z$ directions. If acceleration in $x$, $y$, and $\mathrm{z}$ directions are $\mathrm{A}_{x}, \mathrm{~A}_{y}, \mathrm{~A}_{z}$ then the resultant will be

$$
A_{G T}=\sqrt{\left(A_{x}^{2}+A_{y}^{2}+A_{z}^{2}\right)}
$$

Thus we got a vector of 51 values of accelerometer resultant

2. Variance (var) - We have calculated variance of all 51 values. It was observed that during fall events, the resultant accelerometer value changes suddenly. Variance is an effective statistical method to measure sudden changes. The following formula has been used for calculating values.

$$
\begin{aligned}
& \text { var }=\frac{1}{N} \sum_{i=1}^{N}(X i-\mu)^{2} \\
& X i: \text { individual data point } \\
& u: \text { mean of data points } \\
& N: \text { total no of data points }
\end{aligned}
$$

3. Standard deviations(std) - Standard deviation is a useful measure that measures how much all the data is spread around the mean. It has been calculated using the following formula

$$
s t d=\sqrt{\frac{1}{N} \sum_{i=1}^{N}(X i-\mu)^{2}}
$$

4.Root Mean Square $\left(\mathbf{E}_{R M S}\right)$ - Root mean square is a very important feature of accelerometer signal. It is the square root of the mean square of data items. It is also a measure of the energy contained in the signal.

$$
E_{R M S}=\sqrt{\frac{x_{0}^{2}+x_{1}^{2}+\ldots+x_{N-1}^{2}}{N}}
$$

5. Euclidean norm (En) - Norm is a function that strictly assigns positive values to each vector. Euclidean norm of the signal is the square root of the sum of the square of the discrete data value of the signal. The Euclidean norm of the n-dimensional vector is as follows

$$
\|x\|=\sqrt{\sum_{i=1}^{n} x_{i}^{2}}
$$


Now we have a vector of 55 values for each ADL or fall activity vector. We have analyzed each vector of ADL or fall activity containing 55 values using one-class classification approach

One class classification based on bagging classifier - In the proposed method one class classification method has been used. In one class classification, only target data is considered in one class, and data other than the target class is considered as an outlier. In this experiment, one class classifier has been used which classifies the data using a bagging classifier ${ }^{(40)}$. A bagging predictor is a method that generated multiples predictors and finally uses them to get an aggregated vector. In this method, the dataset is divided into test $\operatorname{set}(\check{\mathrm{T}})$ and a learning $\operatorname{set}(\bigotimes)$, and a classification tree was constructed with a learning set $(\bigotimes)$. A bootstrap sample is selected from the learning set $(\mathbb{\otimes})$ and the tree is grown using bootstrap sample and 10 fold cross-validation. We have used bag size percentage -100 and bag size 100 with seed value 100 .

In this experiment, data of only ADL activities have been used to train the classifier, and ADL and fall activities have been used to test the classifier. ADL activities are considered as target and fall activities as considered as outlier. When we test the model, ADL activities are classified as targets, and fall activities are considered as unknown instances.

\section{Results and discussion}

The proposed model is tested on both datasets. In Medrano et. al. ${ }^{(22)}$ data were collected in two modes first when the mobile is in the pocket and second when the mobile is in the handbag. We build the one-class classification model in two ways. (1) Build the model using only 51 resultant values (without features). (2) Build the model using 55 values (with features)

We test the model in both types of data i.e. ADL activities and fall activities. We also test the model in the Mobifall dataset containing eight types of falls. Following are the results when we test the model on both datasets i.e. Medrano et. al. ${ }^{(35)}$ dataset and Mobifall dataset.

Table 1. Result when we test the model onMedrano et al. ${ }^{(35)}$ dataset

\begin{tabular}{|c|c|c|c|c|c|}
\hline \multirow[t]{2}{*}{ S.No } & \multirow[t]{2}{*}{ Performance measure } & \multicolumn{2}{|c|}{$\begin{array}{l}\text { When a classification model is built without } \\
\text { features }\end{array}$} & \multicolumn{2}{|c|}{$\begin{array}{l}\text { When a classification model is built with } \\
\text { features }\end{array}$} \\
\hline & & $\begin{array}{l}\text { When smart phone is } \\
\text { in pocket }\end{array}$ & $\begin{array}{l}\text { When smart phone } \\
\text { is in handbag }\end{array}$ & $\begin{array}{l}\text { When smart phone } \\
\text { is in pocket }\end{array}$ & $\begin{array}{l}\text { When smart phone is } \\
\text { in handbag }\end{array}$ \\
\hline 1 & Sensitivity & 100.0000 & 100.0000 & 100.0000 & 100.0000 \\
\hline 2 & Specificity & 88.8690 & 90.7112 & 94.9207 & 93.6139 \\
\hline 3 & Accuracy & 89.5420 & 92.5869 & 95.2278 & 94.9035 \\
\hline 4 & Precision & 36.6351 & 73.1469 & 55.8889 & 79.8473 \\
\hline 5 & F1 score & 53.6247 & 84.4911 & 71.7035 & 88.7946 \\
\hline 6 & G Mean & 94.2703 & 95.2424 & 97.4272 & 96.7543 \\
\hline 7 & Critical Success Index(CSI) & 36.6351 & 73.1469 & 55.8889 & 79.8473 \\
\hline 8 & $\begin{array}{l}\text { Matthews correlation coefficient } \\
\text { (MCC) }\end{array}$ & 57.0590 & 81.4570 & 72.8355 & 86.4571 \\
\hline 9 & Bookmaker Informedness (BM) & 187.8690 & 189.7112 & 193.9207 & 192.6139 \\
\hline
\end{tabular}

Table 2. Result when we test the model on Mobifall dataset

\begin{tabular}{llll}
\hline \multirow{2}{*}{ S.No } & Activity & Fall prediction accuracy & \\
\cline { 3 - 4 } & & $\begin{array}{l}\text { Bagging based one-class classification on } \\
\text { the dataset without features. }\end{array}$ & $\begin{array}{l}\text { Bagging based one-class classification } \\
\text { on the dataset with features. }\end{array}$ \\
\hline 1 & Forward lying (FOL) fall & $100 \%$ & $100 \%$ \\
2 & Front knee lying (FKL) fall & $100 \%$ & $100 \%$ \\
3 & Back sitting chair (BSC) fall & $100 \%$ & $100 \%$ \\
4 & Sideward lying (SDL) fall & $100 \%$ & $100 \%$ \\
\hline
\end{tabular}

From Table 1 we can see that when we test bagging one class classification based fall detection model which was built using only 51 resultant accelerometer values in medrano et al. dataset ${ }^{(35)}$, about 6946 instances out of 7816 (approx $\left.88.86 \%\right)$ instances when mobile is in the pocket were correctly classified as ADL activities and 1875 instances out of 2067 (approx $90.7 \%$ ) instances when mobile is in handbag were correctly classified as ADL activities. When the model was tested on fall activities, 503 instances 
out of 503 (approx $100 \%$ ) instances when mobile is in the pocket were correctly classified as fall activities and 523 instances out of 523 (approx 100\%) instances when mobile is in handbag were correctly classified as fall activities. When the same model was tested on the Mobifall dataset (as shown in Table 2) all four types of fall Forward lying (FOL) fall, Front knee lying (FKL) fall, Back sitting chair (BSC) fall, Sideward lying (SDL) fall were correctly classified as outlier i.e. fall.

From Table 1 we can see that when we test one class classification based fall detection model which was built using only 51 resultant accelerometer values and four calculated features (Variance, Standard Deviation, Root Mean Square, Euclidean Norm) in medrano et. al. dataset ${ }^{(35)}$, about 7419 instances out of 7816 (approx 94.9\%) instances when mobile is in the pocket were correctly classified as ADL activities and 1935 instances out of 2067 (approx 93.6\%) instances when mobile is in handbag were correctly classified as ADL activities. When the model was tested on fall activities, 503 instances out of 503 (approx 100 $\%$ ) instances when mobile is in the pocket were correctly classified as fall activities and 523 instances out of 523 (approx 100\%) instances when mobile is in handbag were correctly classified as fall activities. When the same model was tested on the Mobifall dataset (as shown in Table 2) all fall activities were correctly classified as outlier i.e. fall.

We have also studied the effect of each feature on the performance of one class classification model as shown in Tables 3 and 4 and in Figure 4

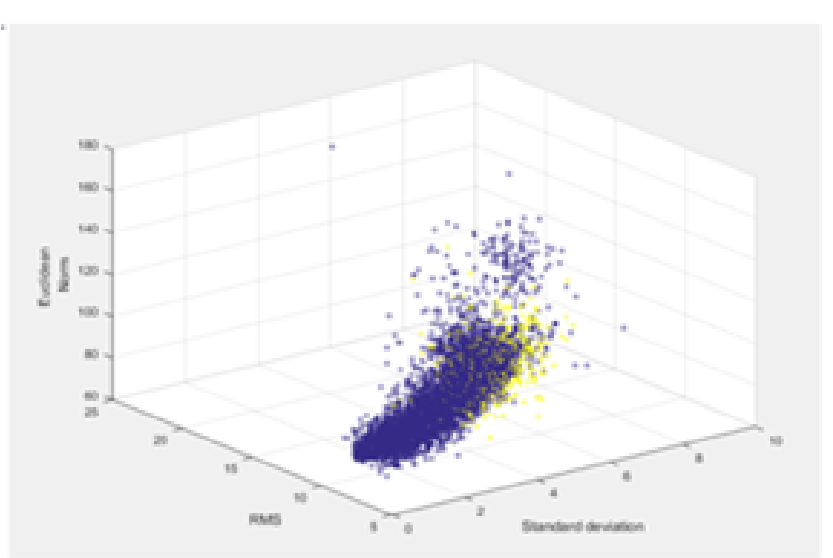

(a) Feature- Std, RMS, En

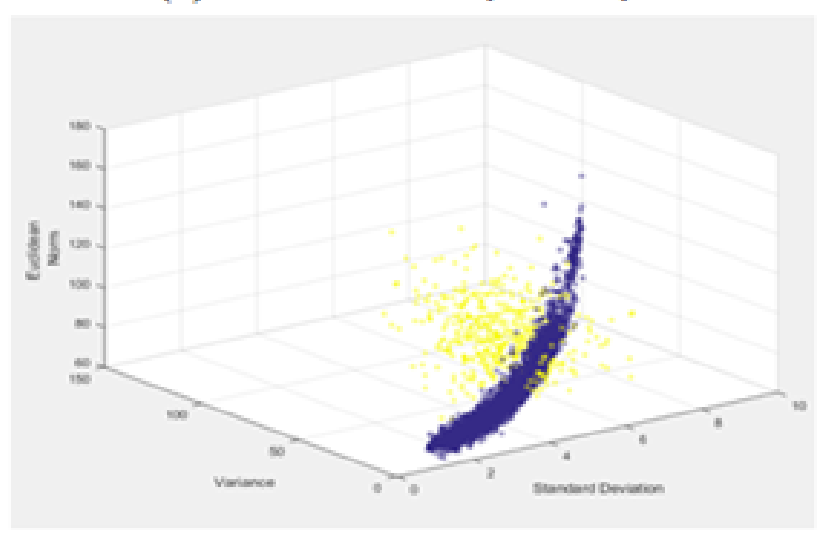

(c) Feature- Std, Var, En

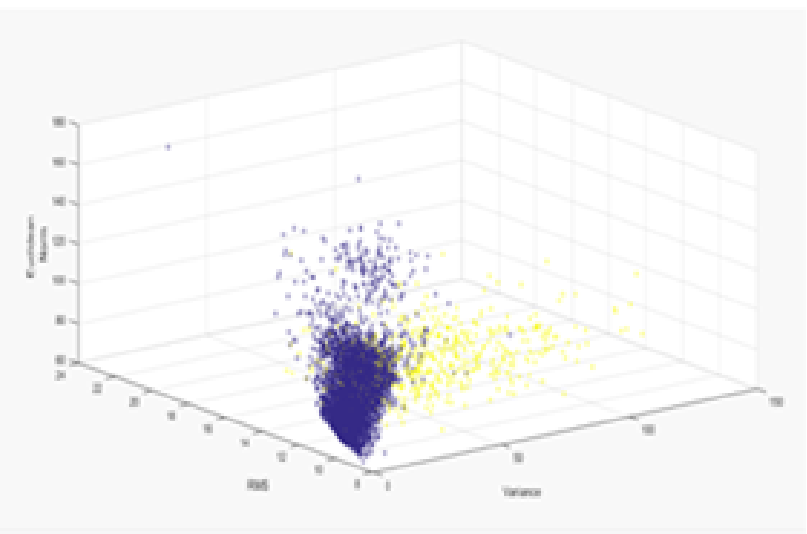

(b) Feature- Var , RMS, En

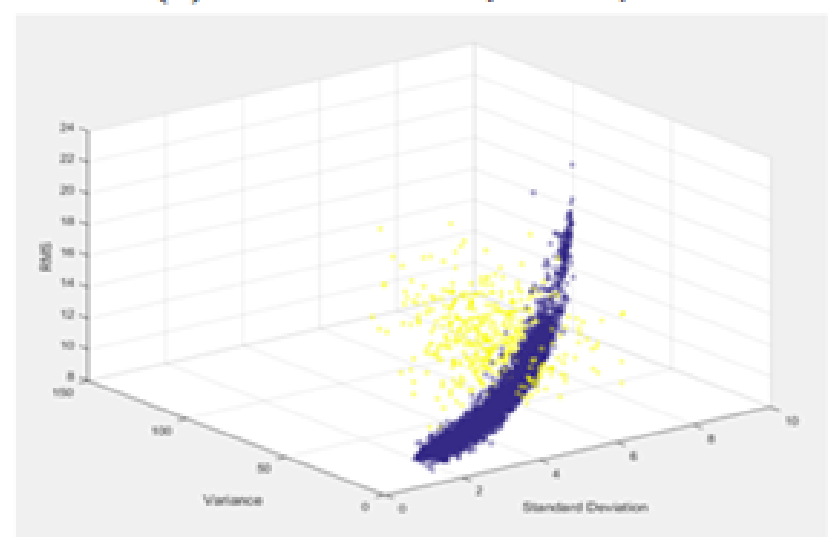

(d) Feature- Std , Var, RMS $\mathrm{ADL}$ activities

\section{Fall activities}

Fig 4. Scatter graph of features 
Table 3. Effect of Standard deviation and variance on one-class classification based fall detection model

\begin{tabular}{|c|c|c|c|c|c|}
\hline \multirow[t]{2}{*}{ S.No } & \multirow[t]{2}{*}{ Performance measure } & \multicolumn{2}{|c|}{$\begin{array}{l}\text { Features used to build the model } \\
\text { Std, RMS, En }\end{array}$} & \multicolumn{2}{|c|}{$\begin{array}{l}\text { Features used to build the model } \\
\text { Var, RMS, En }\end{array}$} \\
\hline & & $\begin{array}{l}\text { When smartphone } \\
\text { is in pocket }\end{array}$ & $\begin{array}{l}\text { When smartphone } \\
\text { is in handbag }\end{array}$ & $\begin{array}{l}\text { When smartphone } \\
\text { is in pocket }\end{array}$ & $\begin{array}{l}\text { When smartphone } \\
\text { is in handbag }\end{array}$ \\
\hline 1 & Sensitivity & 100.0000 & 100.0000 & 100.0000 & 100.0000 \\
\hline 2 & Specificity & 88.5235 & 90.1306 & 94.4857 & 93.3237 \\
\hline 3 & Accuracy & 89.2175 & 92.1236 & 94.8191 & 94.6718 \\
\hline 4 & Precision & 35.9286 & 71.9395 & 53.8544 & 79.1225 \\
\hline 5 & F1 score & 52.8639 & 83.6800 & 70.0070 & 88.3446 \\
\hline 6 & G Mean & 94.0869 & 94.9371 & 97.2037 & 96.6042 \\
\hline 7 & Critical Success Index(CSI) & 35.9286 & 71.9395 & 53.8544 & 79.1225 \\
\hline 8 & $\begin{array}{l}\text { Matthews correlation coefficient } \\
\text { (MCC) }\end{array}$ & 56.3961 & 80.5230 & 71.3335 & 85.9302 \\
\hline 9 & Bookmaker Informedness (BM) & 187.5235 & 189.1306 & 193.4857 & 192.3237 \\
\hline
\end{tabular}

Table 4. Effect of Euclidean norm (En) and Root Mean Square (RMS) on one class classification based fall detection model

\begin{tabular}{llllll}
\hline \multirow{2}{*}{ S.No Performance measure } & \multirow{2}{l}{$\begin{array}{l}\text { Features used to build the model } \\
\text { Std, Var, En }\end{array}$} & \multicolumn{2}{l}{$\begin{array}{l}\text { Features used to build the model Std, Var, } \\
\text { RMS }\end{array}$} \\
\cline { 3 - 6 } & & $\begin{array}{l}\text { When smart } \\
\text { phone is in pocket }\end{array}$ & $\begin{array}{l}\text { When smart phone } \\
\text { is in handbag }\end{array}$ & $\begin{array}{l}\text { When } \begin{array}{l}\text { smart } \\
\text { phone is in pocket }\end{array} \\
\text { When smart phone } \\
\text { is handbag }\end{array}$ \\
\hline 1 & Sensitivity & 100.0000 & 100.0000 & 100.0000 & 100.0000 \\
2 & Specificity & 94.9335 & 93.6139 & 94.9335 & 93.6139 \\
3 & Accuracy & 95.2398 & 94.9035 & 95.2398 & 94.9035 \\
4 & Precision & 55.9511 & 79.8473 & 55.9511 & 79.8473 \\
5 & F1 score & 71.7546 & 88.7946 & 71.7546 & 88.7946 \\
6 & G Mean & 97.4338 & 96.7543 & 97.4338 & 96.7543 \\
7 & Critical Success Index(CSI) & 55.9511 & 79.8473 & 55.9511 & 79.8473 \\
8 & Matthews correlation coefficient (MCC) & 72.8809 & 86.4571 & 72.8809 & 86.4571 \\
9 & Bookmaker Informedness (BM) & 193.9335 & 192.6139 & 193.9335 & 192.6139 \\
\hline
\end{tabular}

From Table 3 we see that removal of variance from the process of building one class classification model seriously affects the performance. From Table 4 we can see that removal of any one of the Root mean square(RMS) feature and Euclidean Norm(En) feature does not affect the model. We got the same results when we exclude Euclidean norm or Root Mean Square (RMS) from the set of features to build one class classification-based fall detection model. From both above Tables (Tables 3 and 4) we can conclude that the variance feature plays a very important role in classifying ADL activities from fall activities and removal of variance, the degraded performance of the model.

We have also compared the classification of the dataset using one-class classification and one-class support vector machine classification $^{(41)}$ as shown in Table 5

Table 5. Comparison of One class classification based on bagging classifier and one-class classification based on support vector machine.

\begin{tabular}{llll}
\hline S.No. & Measures for comparison for one-class classification & Bagging classifier & SVM classifier \\
\hline 1 & Number of training instances & 8894.70 & 8894.70 \\
2 & Number of Testing instances & 988.30 & 988.30 \\
3 & Percentage of correctly classified instances & 88.27 & 11.51 \\
4 & Percentage of incorrectly classified instances & 0 & 0 \\
5 & Percentage of unclassified instances & 11.73 & 88.49 \\
6 & True positive & 1 & 1 \\
7 & False negative & 0 & 0 \\
8 & Time for training & 159.96 & 131.25 \\
9 & Time for testing & 0.01 & 3.41 \\
\hline
\end{tabular}


From the results, it can be seen that percentage of correctly classified instances are $88.27 \%$ and the percentage of incorrectly classified instances are $11.73 \%$ while building the model using one-class classification based on bagging classifier and correctly classified instances are $11.57 \%$ and the percentage of incorrectly classified instances are $88.49 \%$ while building the model using a one-class classification based on support vector machine. From Table 5, it can be seen that the bagging classifier performs much better than the support vector machine classifier.

\section{Conclusion}

Human falls can be detected based on the approach that its accelerometer data during fall is different than accelerometer data of normal living activities. Two publically available fall detection datasets were used in this method. The PKI descritize filter has been applied for filtering accelerometer data. Five different features were calculated on that dataset for classification. After that one class classification was used to build the model because real datasets of fall activities are not available, all fall datasets are simulated in lab. It is found that that proposed method gave very good results on both datasets i.e. all ADL activities were correctly classified as the target and all fall activities were correctly classified as an outlier. This one-class classification is very important because it can be effectively applied in real-life fall. In this proposed method, it is also found that variance is an important feature while building a fall detection model. Although this work is tested on a limited number of the dataset but in the future, this work can also be extended by incorporating more datasets in the study to validate its reliability on a large population.

\section{References}

1) Rubenstein LZ. Falls in older people: epidemiology, risk factors and strategies for prevention. Age Ageing. 2006;35(2):37-41. Available from: https://doi.org/10.1093/ageing/afl084.

2) Falls. 2020. Available from: https://www.who.int/news-room/fact-sheets/detail/falls.

3) Gia T, Tcarenko I, Sarker V, Rahmani A, Westerlund T, Tenhunen H. IoT-based fall detection system with energy efficient sensor nodes. NORCAS 2016 2nd IEEE NORCAS Conference. 2016.

4) Yacchirema D, Puga JSD, Palau C, Esteve M. Fall detection system for elderly people using IoT and ensemble machine learning algorithm. Personal and Ubiquitious compting. 2019;23:801-817. Available from: https://doi.org/10.1007/s00779-018-01196-8.

5) Mubashir M, Shao L, Seed L. A survey on fall detection: Principles and approaches. Neurocomputing. 2013;100:144-152. Available from: https: //www.sciencedirect.com/science/article/abs/pii/S0925231212003153.

6) Tax D. One-class classification. 2001. Available from: https://ci.nii.ac.jp/naid/10010287825/.

7) Khan SS, Madden MG. A survey of recent trends in one class classification. Lecture Notes in Computer Science (including subseries Lecture Notes in Artificial Intelligence and Lecture Notes in Bioinformatics). 2010;6206. Available from: https://doi.org/10.1007/978-3-642-17080-5_21.

8) Moya MM, Koch MW, Hostetler LD, Moya MM, Koch MW, Hostetler LD. One-class classifier networks for target recognition applications. STIN. 1993;93:24043. Available from: https://ui.adsabs.harvard.edu/abs/1993STIN...9324043M/abstract.

9) Ritter G, Gallegos MT. Outliers in statistical pattern recognition and an application to automatic chromosome classification. Pattern Recognition Letters. 1997;18(6):525-539. Available from: https://www.sciencedirect.com/science/article/abs/pii/S0167865597000494.

10) Bishop CM. Novelty detection and neural network validation. IEEE Proceding of confrence on Vision. 1994;141(4):217-222. Available from: https: //ieeexplore.ieee.org/document/318023/.

11) Japkowicz N. Concept-learning in the absence of counter-examples: an autoassociation-based approach to classification. 1999;p. 169. Available from: https://doi.org/10.7282/T3J96B11.

12) Hempstalk K, Frank E, Witten IH. One-Class Classification by Combining Density and Class Probability Estimation. In: Machine Learning and Knowledge Discovery in Databases. Springer. ;p. 505-519. doi:http://link.springer.com/10.1007/978-3-540-87479-9_51.

13) Sarkar P, Sinha D. Application on Pervasive Computing in Healthcare - A Review. Indian Journal of Science and Technology. 2017;10(3):1-10. doi:10.17485/ijst/2017/v10i3/110619.

14) Tolkiehn M, Atallah L, Lo B, Yang GZ. Direction sensitive fall detection using a triaxial accelerometer and a barometric pressure sensor. Proceedings of the Annual International Conference of the IEEE Engineering in Medicine and Biology Society. 2011;p. 369-372. Available from: https://pubmed.ncbi.nlm. nih.gov/22254325/.

15) Fang SH, Liang YC, Chiu KM. Developing a mobile phone-based fall detection system on Android platform. 2012 Computing, Communications and Applications Conference. 2012;p. 143-146. Available from: http://ieeexplore.ieee.org/document/6154019/.

16) S KE, Rao KR. Implementation of Cloud IoT - Based Patient Fall Detection System with GeoTagging using Tilt Sensors and Texas Instruments' CC3200. Indian Journal of Science and Technology. 2019;12(34):1-7. doi:10.17485/ijst/2019/v12i34/147071.

17) Ryu JT, Moon BH. Fall Detection Algorithm based on Peaks of Voltage Measurements from the Accelerometer. Indian Journal of Science and Technology. 2016;9(45):1-6. doi:10.17485/ijst/2016/v9i45/106768.

18) Prince PGK, Hemamalini R, Rajkumar RI. LabVIEW based Abnormal Muscular Movement and Fall Detection using MEMS Accelerometer during the Occurrence of Seizure. Indian Journal of Science and Technology. 2014;7(10):1625-1631. doi:10.17485/ijst/2014/v7i10.9.

19) Albert MV, Kording K, Herrmann M, Jayaraman A. Fall Classification by Machine Learning Using Mobile Phones. Plos One. 2012;7(5):e36556. Available from: https://dx.plos.org/10.1371/journal.pone.0036556.

20) Shibuya N, Nukala BT, Rodriguez AI, Tsay J, Nguyen TQ, Zupancic S. A real-time fall detection system using a wearable gait analysis sensor and a Support Vector Machine (SVM) classifier. 2015 8th International Conference on Mobile Computing and Ubiquitous Networking. 2015;p. 66-67. Available from: https://ieeexplore.ieee.org/document/7061032. 
21) Wang J, Zhang Z, Li B, Lee S, Sherratt R. An enhanced fall detection system for elderly person monitoring using consumer home networks. IEEE Transaction on consumer electronics. 2014;60(1):23-29. Available from: https://ieeexplore.ieee.org/document/6780921.

22) Huynh QT, Tran BQ. Time-Frequency Analysis of Daily Activities for Fall Detection. Signals. 2021;2021(2):1-12. Available from: https://www.mdpi. com/2624-6120/2/1/1/htm.

23) Liu N, Zhang D, Su Z, Wang T. Preimpact Fall Detection for Elderly Based on Fractional Domain. Mathematical Problem in Engineering. 2021. Available from: https://www.hindawi.com/journals/mpe/2021/6661034/.

24) Panhwar M, Shah SMS, Shah SMZS, S SMZA, Chowdhry BS. Smart Phone Based Fall Detection using Auto Regression Modeling in a Non-Restrictive Setting. Indian Journal of Science and Technology. 2017;10(5):1-6. doi:10.17485/ijst/2017/v10i5/111274.

25) Raghu G, Ponraj AS. Remote Safety Assistance and Health Monitoring System. Indian Journal of Science and Technology. 2016;9(45):1-6. doi:10.17485/ijst/2016/v9i45/99611.

26) Madhubala JS, Umamakeswari A. A Vision based Fall Detection System for Elderly People. Indian Journal of Science and Technology. 2015;8(S9):1-9. doi:10.17485/ijst/2015/v8iS9/65545.

27) Bilal M, Fergani B, Fleury A. Integrating Prior Knowledge in Weighted SVM for Human Activity Recognition in Smart Home. Lecture Notes in Computer Science (including subseries Lecture Notes in Artificial Intelligence and Lecture Notes in Bioinformatics);2017:233-239. Available from: https://link.springer.com/chapter/10.1007/978-3-319-66188-9_20.

28) Wan Q, Li Y, Li C, Pal R. Gesture recognition for smart home applications using portable radar sensors. 36th Annual International Conference of the IEEE Engineering in Medicine and Biology Society. 2014;p. 6414-6417. doi:10.1109/EMBC.2014.6945096.

29) Alegre F, Amehraye A, Evans N. Evans "A one-class classification approach to generalised speaker verification spoofing countermeasures using local binary patterns. IEEE 6th International Conference on Biometrics: Theory, Applications and Systems. 2013. Available from: https://ieeexplore.ieee.org/abstract/ document/6712706/.

30) Guerbai Y, Chibani Y, Hadjadji B. The effective use of the one-class SVM classifier for handwritten signature verification based on writer-independent parameters. Pattern Recognition Letters. 2015;48(1):103-113. Available from: https://www.sciencedirect.com/science/article/abs/pii/S0031320314002751.

31) Wan M, Shang W, Zeng P. Double Behavior Characteristics for One-Class Classification Anomaly Detection in Networked Control Systems. IEEE transactions on Information Forensics and Security. 2017;12(12):3011-3023. Available from: https://ieeexplore.ieee.org/abstract/document/7987719/.

32) Krawczyk B, Filipczuk P. Cytological image analysis with firefly nuclei detection and hybrid one-class classification decomposition. Engineering Application of Artificial Intelligence. 2014;31:126-135. Available from: https://www.sciencedirect.com/science/article/abs/pii/S0952197613001917.

33) Kemmler M, Rodner E, Denzler J. One-class classification with gaussian processes. Lecture Notes in Computer Science (including subseries Lecture Notes in Artificial Intelligence and Lecture Notes in Bioinformatics. 2011;p. 489-500. Available from: https://www.sciencedirect.com/science/article/abs/pii/ S0031320313002574.

34) Leng Q, Qi H, Miao J, Zhu W, Su G. One-class classification with extreme learning machine. Mathematical Problems in Engineering. 2015. Available from: https://www.hindawi.com/journals/mpe/2015/412957/abs/.

35) Medrano C, Igual R, Plaza I, Castro M. Detecting Falls as Novelties in Acceleration Patterns Acquired with Smartphones. PLoS One. $2014 ; 9(4): 94811$. Available from: https://dx.plos.org/10.1371/journal.pone.0094811.

36) Medrano C, Igual R, García-Magariño I, Plaza I, Azuara G. Combining novelty detectors to improve accelerometer-based fall detection. Medical and Biological Engineering and Computing. 2017;(10):1849-1858. Available from: http://link.springer.com/10.1007/s11517-017-1632-z.

37) Micucci D, Mobilio M, Napoletano P, Tisato F. Falls as anomalies? An experimental evaluation using smartphone accelerometer data. Journal of Ambient Intelligent and Humanized Computing. 2017;8(1):87-99. Available from: http://link.springer.com/10.1007/s12652-015-0337-0.

38) Sheikh SY, Jilani MT. A ubiquitous wheelchair fall detection system using low-cost embedded inertial sensors and unsupervised one-class SVM. Journal of Ambient Intelligent and Humanized Computing. 2021;2021:1-16. Available from: https://link.springer.com/article/10.1007/s12652-021-03279-6.

39) Vavoulas G, Pediaditis M, Spanakis EG, Tsiknakis M. The MobiFall dataset: An initial evaluation of fall detection algorithms using smartphones. 13th IEEE International Conference on BioInformatics and BioEngineering. 2013. Available from: https://www.sciencedirect.com/science/article/abs/pii/ S0031320313002574.

40) Breiman L. Bagging predictors. Machine Learning. 1996;24(2):123-140. Available from: https://link.springer.com/article/10.1007/BF00058655.

41) Chang CC, Lin CJ. LIBSVM: A Library for support vector machines. ACM Transaction on Intelligent System and Technology. 2011;2(3). Available from: https://dl.acm.org/doi/abs/10.1145/1961189.1961199. 